\title{
Distribution Pattern of Pudendal Nerve Plexus for the Phallus Retractor Muscles in the Cock
}

\author{
By \\ ${ }^{{ }^{11}}$ Kazutaka OHSAWA, ${ }^{{ }^{2} 2}$ Takao NISHIDA, Masamichi KUROHMARU and \\ Yoshihiro HAYASHI
}

\author{
Department of Veterinary Anatomy, Faculty of Agriculture, \\ University of Tokyo, 1-1-1, Yayoi, Bunkyo-ku, Tokyo 113, Japan \\ - Received for Publication, October 19, 1990-
}

\begin{abstract}
Key Words: Pudendal plexus, Phallus retractor muscle, Pudendal nerve, Intermediate caudal nerve, Chicken
Summary: Two paired retractor muscles, $m$. retractor phalli cranialis (MRPCr) and $m$. retractor phalli caudalis (MRPCa) exist as the intrinsic cloacal muscles of the chicken. Pudendal plexus was formed by the ventral rami of roots 30-34. Pudendal nerve (PN) and the intermediated caudal nerve (ICN) were composed of twigs from roots $30-33$ and roots 30-34, respectively. Two or three rootlets participated in the PN; its third one originated from the root 33 which is the first root of caudal trunk in pudendal plexus. The non-striated MRPCr was innervated by the PN, whereas the striated MRPCa by the ICN.
\end{abstract}

Two paired retractor muscles exist within the intrinsic cloacal muscles of birds, being $m$. retractor phalli cranialis (synonyms: $m$. dilator cloacae) and $m$. retractor phalli caudalis (synonyms: $m$. levator cloacae and $m$. retractor phalli) (Liebe, 1914; Knight, 1970). By histological examination, it has been demonstrated that the cranial muscle (MRPCr) and the caudal muscle (MRPCa) are a non-striated (smooth) muscle and a striated muscle, respectively, in the drake (Liebe, 1914; Rautenfeld et al., 1974), cock (Nishiyama, 1955; Ohsawa et al., 1989) and pigeon (Baumel, 1988). Vanden Berge (1979) has stated that these muscles are of infrequent occurrence, suggesting the possibility that those have only a minor functional role. However, the existence of both muscles is confirmed in all the chicken specimens observed (Ohsawa et al., 1989; 1990a).

In addition, sexual dimorphism of the MRPCr was observed in the thickness of muscle-cell bundle and the quantity of connective tissue (Ohsawa et al., 1989). The functional significance of the retractor muscles is also suggested by observations of their traveling routes, i.e., the principal bundle of the MRPCr is inserted into the basis of the median phallic body and the MRPCr arrives more laterally at the cloacal wall or near the lymphatic folds (Liebe, 1914).

Although it is presumed that the MRPCa may accept the intermediate caudal nerve in the pigeon (Baumel, 1988), there is no evidence on the nerve supply in the chicken. In the present report, we deal with the course and distribution of the nerve supplied to both the phallus retractor muscles.

\section{Materials and Methods}

Six adult male White Leghorn chickens, weighing about $3 \mathrm{~kg}$, were used in the present study. The animals were sacrificed by bloodletting under the anesthesia with sodium pentobarbital and fixed in $10 \%$ formalin for two days. After the decalcification with $15 \%$ nitric acid for 5 to 7 days, fat of the specimens was removed by solid ethanol for three days. Then, the specimens were subjected to gross anatomy for clarifying the course and distribution of the pudendal plexus, the sacral sympathetic nerves and cloacorectal part of intestinal nerve.

\section{Results and Discussion}

The pudental plexus is formed by the ventral rami of five spinal nerve (roots 30-34) just behind the

*1) Present address: Torii Nutrient-stasis project, Exploratory Research for Advanced Technology, Research Development Corporation of Japan. Technowave 100, 1-1-25, Shin-Urashima-cho, Kanagawa-ko, Yokohama 221, Japan.

*2) Present address: Laboratory of Anatomy and Physiology, Department of Animal Husbandry, College of Agriculture and Veterinary Medicine, Nihon University, Kameino 1866, Fujisawa, Kanagawa 252 Japan. 
sacral plexus. The plexus is roughly divided into the cranial trunk (roots 30-32) and the caudal trunk (roots 33-34); the former gives rise to the lateral caudal nerve $(\mathrm{LCN})$ and the pudendal nerve $(\mathrm{PN})$, and the latter gives an origin to the intermediate caudal nerve (ICN). These observations correspond with those by Baumel (1975).

It has definitely been shown by detailed observations that the $\mathrm{PN}$ and the $\mathrm{LCN}$ are composed of twigs from roots $30-33$ and the $\mathrm{ICN}$ is made up of those from roots 30-34. Two or three original rootlets from roots 30-33 join the PN on the dorsal surface of proximal part of ureter. Both the first and second rootlets of $\mathrm{PN}$ originate from the cranial trunk (roots 30-32), while the third rootlet, when it is present, spring from the caudal trunk, especially from the root 33 . The third rootlet was not observed in two specimens and it remains unclear whether it was due to the technical error or the variations. There are a few reports (Knight, 1970; Watanabe, 1972; Baumel, 1975) on the distribution of peripheral nerve arising from the pudendal plexus. These results are generally in agreement with our observations including two minor differences; first, the PN originated from roots $30-33$ in our observations, although Knight (1970) mentioned the contribution of root 34 to the nerve, and secondly, Watanabe (1972) and Baumel (1975) did not observed a small anastomosis between the cranial and caudal trunks.

Recently, the detailed topography and distribution of the pigeon pudendal plexus have been reported by Baumel (1988). The plexus begins with roots $27-31$ in the pigeon, whereas it originates with roots $30-34$ in the chicken examined in this study. It is thought that there is no essential difference between the two species, because it is probably due to the existence of three more cervical nerves in the chicken. The $\mathrm{PN}$ was reported to arise from the pudendal plexus by one or two rootlets in the pigeon. When the second rootlet was present, it arose from the caudal trunk (3rd or 4th root) of the plexus (Baumel, 1988). There is no inconsistency between his result and our observation, by taking a view that the final rootlet of PN may arise from the caudal trunk of pudendal plexus in both the pigeon and chicken.

The PN send extremely fine branch to the MRPCr. The nerve is believed to contain parasympathetic preganglionic nerve fibers and sympathetic postganglionic nerve fibers (a personal communication with Dr. Ohmori of Nagoya University). By the injection of true blue into the MRPCr, many bluefluorescent nerve cells were observed in the cloacal ganglia in the PN on the dorsal side of distal ureter (Ohsawa et al., 1990b). This histological tracer experiment also demonstrates the PN controlling the MRPCr

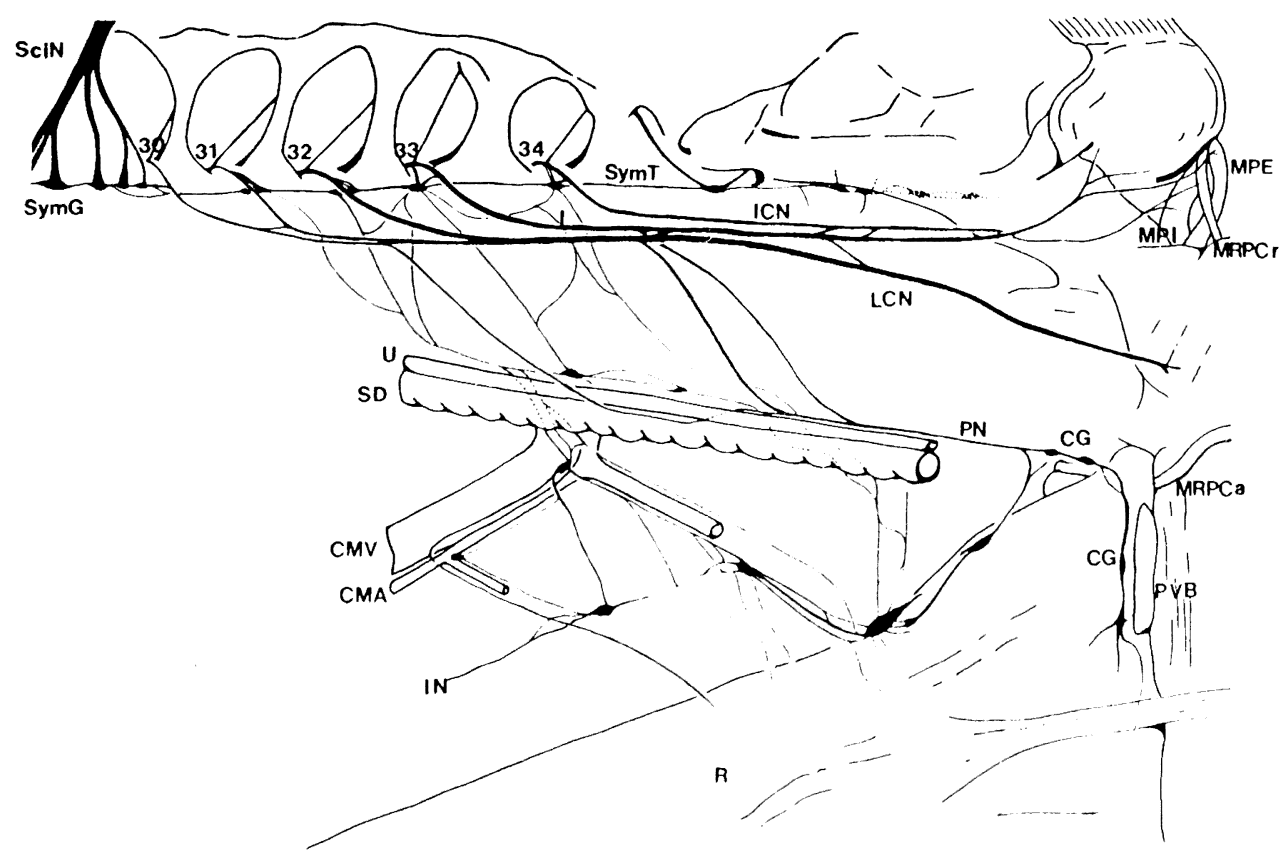

Fig. 1. Pudendal plexus and its distribution in the cock. Ventro lateral view. The plexus is derived from ventral rami of spinal nerves 30-34. The $m$. retractor phalli cranialis (MRPCr) and m. retractor phalli caudalis (MRPCa) are supplied with the pudendal nerve (PN) and intermediate caudal nerve (ICN) respectively. The $\mathrm{PN}$ and the intestinal nerve (IN) are connected by the fine branch and the ganglia. Abbreviations; CMA, caudal mesenteric artery; CMV, caudal mesenteric vein; CG, cloacal ganglia; LCN, lateral caudal nerve; MPE, m. pubocaudalis externus; MPI, m. pubocaudalis internus; PVB, paracloacal bascular body; R, rectum; SciN, sciatic nerve; SD, seminal duct; SymG, sympathetic ganglion; SymT, sympathetic trunk; U, ureter. 
and the existence of many MRPCr-innervating parasympathetic postganglionic nerve cells in the terminal part of the PN or in the vicinity of the muscle.

A nerve branch of the ICN, especially ramus from root 34, penetrates the MRPCa. After the true blue injection into the MRPCa, the true blue-positive nerve fiber-like structures were observed throughout ramus of root 34 (Ohsawa et al., 1990b). These results suggest that many somatic motor nerve fibers pass along the ICN (root 34) and then innervate the striated MRPCa. Baumel (1988) has not verified but has expected that the MRPCa in the pigeon accepts the somatic ICN. According to our results in the chicken, the pigeon MRPCa may be also innervated by the nerve fibers from the ICN.

The autonomic nerve is supplied on the MRPCr via the pudendal and/or intestinal nerve because the muscle, as well as the walls of rectum, ductus deferens and ureter, is composed of smooth muscle cells. Furthermore, four types of axon varicosity, namely, cholinergic, noradrenergic, aminergic and peptidergic ones, are observed in nerve endings of the chicken MRPCr (Ohsawa, 1989). This result indicates that the MRPCr is controlled by the sympathetic, parasympathetic and non-adrenergic non-cholinergic (NANC) nerve, and that the pudendal nerve stands between the parasympathetic nerve cell and the MRPCr.

However, origins and courses of sympathetic and NANC nerves are not identified in the MRPCr. The anastomosing branches from the pudendal nerve join with the intestinal nerve, mediating the ganglia on the dorsal wall of cloaca (Watanabe, 1972). It is believed that the cloacal part of intestinal nerve in the chicken is a sympathetic nerve, and the nerve may penetrate the MRPCr via both the pudendal and intestinal nerves.

In digestive tract the NANC nerve fibers, such as those immunopositive with substance $\mathrm{P}$, vasoactive intestinal polypeptide and serotonin, are derived from intrinsic nerve cells in the submucosal and the myenteric plexuses (Gershon, 1977; Schultzberg, 1980). Because nerve cells are not observed in the MRPCr, the NANC nerve fibers can intrinsically be originated in the cloacal ganglia instead of the plexuses.

\section{References}

1) Baumel, J. J.: Aves nervous system. In "Sisson and Grossman's The anatomy of the domestic animals" (Getty, R. ed.), vol. 2. Saunders, Philadelphia, pp.2019-2062, 1975.

2) Baumel, J. J.: Functional morphology of the tail apparatus of the pigeon (Columba livia). Springer-Verlag, Berlin. 1988.

3) Gershon, M. D., Dreyfus, C. F., Pickel, V. M., Joh, T. H. and Reis, D. J.: Serotonergic neurons in the peripheral nervous system: Identification in gut by immunohistochemical localization of tryptophan hydroxylase. Proc. Natl. Acad. Sci. USA, 74: 3086-3089, 1977.

4) Knight, C. E.: The anatomy of the structures involved in the erection-dilution mechanism in the male domestic fowl. Ph.D. thesis, Michigan State Univ., 1970.

5) Liebe, W.: Das männliche Begattungsorgan der Hausente. Jen. Z. Med. Naturw., 51: 627-696, 1914.

6) Nishiyama, H.: Studies on the accessory reproductive organs in the cock. Jour. Fac. Agric., Kyushu Univ., 10: 277-305, 1955.

7) Ohsawa, K., Nishida, T., Kurohmaru, M. and Hayashi, Y.: Ultrastructural identification of axons supplying the $m$. retractor phalli cranialis in chickens. Okajimas Folia Anat. Jpn., 66: 23-38, 1989.

8) Ohsawa, K., Nishida, T., Kurohmaru, M. and Hayashi, Y .: Distribution of acetylcholinesterase in the nerve endings of chicken $m$. retractor phalli cranialis. J. Anat., 170: 63-70, 1990a.

9) Ohsawa, K., Nishida, T., Hayashi, Y. and Kurohmaru, M.: The innervation of retractor phallus muscles in chickens. Acta Anat. Nippon., 65: 337, 1990 b.

10) Rautenfeld, B. D., Preuss, F. and Fricke, W.: Neue Daten zur Erektion und Reposition des Erpelphallus. Der praktische Tierarzt, 10: 553-556, 1974.

11) Schultzberg, M. D., Hökfelt, G., Nilsson, G., Terenius, L., Rehfeld, J. F., Brown, M., Elde, R., Doldstein, M. and Said, S.: Distribution of peptide- and catecholamine-containing neurons in the gastro-intestinal tract of rat and guinea-pig: Immunohistochemical studies with antisera to substance $\mathrm{P}$, vasoactive intestinal polypeptide, enkephalins, somatostatin, gastrin/cholecystokinin, neurotensin and dopamine $\beta$ hydroxylase. Neuroscience, 5: 689-744, 1980.

12) Vanden Berg, J. C.: Myologia, In "Nomina anatomica avium.' (Baumel, J. J., King, A. S., Lucas, A. M., Breazile, J. E. \& Evans, H. E. eds.) Academic Press, London, 1979.

13) Watanabe, T.: Comparative and topographical anatomy of the fowl. LXIV. Sympathetic nervous system of the fowl. Part 2. Nervous intestinalis. Jpn. J. Vet. Sci., 34: 303-313, 1972. 